\section{GESTIÓN DOCENTE EN LA ADMINISTRACIÓN DE EDUCACIÓN A} DISTANCIA

\section{TEACHING MANAGEMENT IN THE ADMINISTRATION OF DISTANCE LEARNING}

\section{Mercedes de la E. Inciarte Rodríguez ${ }^{1}$.} Universidad del Zulia.

\section{Recibido: 06-06-07}

\section{RESUMEN}

La presente investigación documental se centra en la importancia de la gestión docente ante la Administración de Educación a Distancia. Se identifican los principales cambios ocurridos en la educación tradicional y la educación a distancia, asi como las exigencias de la gestión del docente ante la administración y facilitación de estudios a distancia en la modalidad virtual. Es así como se plantea las funciones, la gestión y los criterios de desempeño para estar al tanto del campo de aplicación y lo conocimientos del docente administrador de espacios virtuales. Finalmente, se establece que la gestió docente cumple las siguientes funciones: dia gestión docente cumple las siguientes funciones. diseñador y productor de contenidos, motivador y promotor de interés de los participantes en el estudio de las temáticas propuestas, guiar y/o reorientar al alumno en el proceso de aprendizaje, ampliar la información, evaluar el proceso de aprendizaje, así como diseña la instrucción de su curso y las evaluaciones de aprendizaje.

Descriptores: gestión docente, desempeño, educación a distancia

1 Licenciada en Educación. Ciencias Pedagógicas. Área Tecnología Instruccional. Magíster en Informática Educativa. Doctora en Ciencias de la Educación Coordinadora Académica del Municipio Nro 1 Ministerio del Poder Popular para la Educación.Coordinadora de Diseño Instruccional del Sistema de Educación a Distancia de de la Universidad del Zulia. Asesora Curricular de la Escuela de Derecho y Trabajo Social de la Universidad del Z la Profesta de Postgrado de la Universidad de Zulia Profesora de Postgrado de la Universidad de

\section{INTRODUCCIÓN}

La educación actual afronta múltiples retos y uno de ellos es dar respuesta a los profundos cambios sociales, económicos y culturales que se prevén para la llamada «Sociedad de la Información y el Conocimiento» en esta era de la Información y la Comunicación, siendo el Internet el que ha generado un enorme interés en todos los ámbitos de nuestra sociedad y su gracias a su creciente uso con fines educativos se ha convertido en un campo abierto a la reflexión e investigación.

Este fenómeno, denominado globalización, ha caracterizado al mundo contemporáneo y facilitado la consolidación de una sociedad basada en el conocimiento, sustentada en una revolución científica y tecnológica que esta moviendo a pasos agigantados todos los cimientos de la sociedad; lo cual, indudablemente, incide en las transformaciones en los ámbitos, sociales, políticos y económicos, desde esta perspectiva, la educación ha cobrado una nueva dimensión, sobre todo en el subsistema de educación superior, por el potencial que ésta representa ante un mundo que reconoce cada vez más la importancia del conocimiento y la utilización de la alta tecnología, dando como resultado la necesidad de una ampliación de la oferta educativa, con modalidades que además de generar acceso a este subsistema, también se transforme respondiendo a estas demandas de cambio a nivel nacional e internacional

De allí, la importancia del análisis del binomio educación y tecnologías de la información y la comunicación y la capacidad docente para enfrentar estos cambios, respondiendo a la doble faceta de docente e investigador, exigiendo una correcta preparación tanto para la adquisición de conocimientos y actualización, como para el desarrollo de nuevas habilidades y destrezas exigibles en una sociedad en permanente cambio.

Bajo esta perspectiva, los cambios implican diversos factores y actores del proceso educativo, el cual ha de desempeñar un papel clave en este proceso de modernización, ameritando un profundo ejercicio de reflexión, que evidencie la inserción de las tecnologías ligadas a la exigencia de la globalización como un fenómeno cultural, reconociendo la necesidad de investigar más acerca del uso de las TIC, sobretodo en la formación continua del profesorado, especialmente si se quiere renovar genuinamente la educación.

El docente debe estar capacitado en el conocimiento de las características de los entornos tecnológicos, de otra manera no se pueden colocar en la perspectiva de analizar 
las potencialidades de ayuda o andamiaje didáctico que ofrecen las TIC. Este conocimiento permite al docente poder evaluar y utilizar la tecnología en los entornos virtuales o como una herramienta o recurso efectiva y eficazmente para hacer más interactivo sus cursos y el desarrollo de las competencias (conocimientos, habilidades, destrezas y actitudes) de los estudiantes.

Ante esta necesidad, en el presente artículo de naturaleza documental, se presentan las funciones y gestión del docente que diseña, produce material didáctico y administra la educación a distancia, para el logro de aprendizajes más significativos y abordar los cambios existente en educación.

\section{LA EDUCACIÓN A DISTANCIA}

El mundo en el que vivimos se identifica con la dinámica, donde lo que es válido hoy, quizás mañana no tenga el mismo valor, siendo la única constante el cambio mismo. Por tal razón educadores, deben estar preparados para el abordaje de nuevas formas de enseñanza y herramientas que permitan lograr con eficiencia y eficacia, la realización, en algunos casos e innovación en otros, de los procesos instruccionales para acceder al conocimiento.

Es así como, según Rosario, (2006) "La Educación Virtual, es una nueva forma viable de enseñanza que viene a suplir necesidades, precariedades propias de la educación y la tecnología educativa".)

La educación virtual ha venido a cubrir necesidades que durante muchos años ha tenido la educación tradicional, pues la sociedad ha estado inmersa en cambios tecnológicos de gran magnitud, en el cual es cada vez mayor el número de personas de todos los niveles socioeconómicos que precisan formarse con el fin de estar a la par de los cambios. Como lo plantea, Zubrira, (1994). "La sociedad ha cambiado y la escuela actual no responde a sus expectativas".

En el Cuadro 1, se detallan las principales diferencias entre el sistema presencial y el sistema de educación a distancia:
Cuadro 1

\section{Diferencia entre las dos modalidades}

\section{EDUCACIÓN MODALIDAD A DISTANCIA} O SEMI PRESENCIAL

EDUCACIÓN MODALIDAD PRESENCIAL

$>\quad$ El profesor y los estudiantes pueden no estar presentes físicamente en el mismo espacio ni en el mismo tiempo. Para que la comunicación se produzco, es necesario crear elementos mediadores entre el docente y el alumno.

$>\quad$ Elimina la rígida frontera de espacio y tiempo que impone el paradigma de clase tradicional

$>\quad$ Demuestra que los participantes pueden aprender sin estar congregados en el mismo sitio y al mismo tiempo.

Fuente: García Aretio (Nov. 2007, pagina 5)

La diferencia entre la enseñanza presencial y a distancia no esta en el si y el no, presencia y ausencia, contacto presencial con el docente o contacto virtual, contacto personal con compañeros del curso o contacto a través del trabajo colaborativo y cooperativo. Sin lugar a dudas, la diferencia esta en los retos que enfrentan, tanto la educación en sentido general como la educación a distancia en particular, en la adaptación e integración de ambas, de manera útil y operativa, en el nuevo contexto que representa el modelo telemático en la actualidad. Al aplicar, en la práctica, los procedimientos y metodologías propios de la educación a distancia, cambia necesariamente, la estructura tradicional de la institución y en la adecuación de todos los entes involucrados en la modalidad.

\section{Competencias del Docente Actual}

El docente del siglo XXI, posee las siguientes competencias globales:

- Guiará el aprendizaje de los alumnos conforme a determinadas reglas metódicas, tales como las relacionadas con la tutoría, la gestión didáctica y la innovación. 
- Es un agente primordial en el proceso de socialización. Por otra parte como juez evaluador, desempeña una función fundamental de control social

- Satisfacción de las necesidades de autorrealización de los individuos en formación y de sus demandas de bienestar

- Cualidades pedagógicas (Habilidades didácticas, tutorías, técnicas de investigación, conocimientos psicológicos y sociales

- Habilidades instrumentales y conocimientos de nuevos lenguajes y características personales (Madurez, seguridad, autoestima, equilibrio emocional, empatía).

\section{Principales Funciones del Docente Actual}

- Planificar cursos (conocer las características individuales y grupales de los participantes y diagnosticar sus necesidades de formación).

- Diseñar estrategias de enseñanza y aprendizaje que consideren la utilización de tecnologías de la información y la comunicación.

- Buscar y preparar recursos y materiales didácticos.

- Proporcionar información y gestionar el desarrollo de las clases

- Motivar al estudiante.

- Facilitar la comprensión de la asignatura.

- Asesorar en el uso de recursos.

- Orientar la realización de actividades.

- Tutoría (presencial y telemática)

- Evaluar.

- Fomentar actitudes necesarias en la sociedad de la información.

- Trabajo de gestión.

- Formación continúa.

- Contacto con el entorno.

\section{Virtualización de la educación}

La educación es un ente socializadora que impulsa al conocimiento epistemológico a sumergirse, hasta llegar el proceso de dialecticidad. Sin embargo, como ya antecedemos, la virtualidad no tiene límites, ni reglas, es espontáneo y transformador que parte de la virtud humana.
Para Silvio (2000), la virtualización “es un proceso y resultado al mismo tiempo del tratamiento y de la comunicación mediante computadora de datos, informaciones y conocimientos". Consiste "en representar electrónicamente y en forma numérica digital, objetos y procesos que encontramos en el mundo real” (pp. 214). Esta modalidad, se distingue por ciertos principios caracartecrìsticos (Silvio, ob.cit)

1. El problema a resolver consiste en dotar de conocimientos a personas que los necesitan para vivir en sociedad exitosamente.

2. Unas personas proveen un conjunto de condiciones, instrumentos y metodologías para facilitarles a los educandos la tarea de adquirir los conocimientos que necesitan.

3. Los educandos se comunican con los educadores y con otros educandos para intercambiar y compartir conocimientos y construir sus conocimientos propios, en diferentes lugares y tiempos.

4. Los educandos complementan esos conocimientos con otros conocimientos almacenados en una red de centros de información o de bibliotecas distribuidas en todo el mundo.

5. Al final del proceso, los educadores, junto con los educandos, evalúan los conocimientos adquiridos.

De igual forma, este autor menciona que los procesos de enseñanza y aprendizaje son los que se realizan en un aula virtual y el resultado de esa virtualización es lo que se podría llamar campus virtual en cual los espacios básicos se hallarían interrelacionados en una totalidad integrada.

\section{Sistemas Interactivos en la Educación}

Según Bravo (2005), "los sistemas interactivos aparecen en educación como una tecnología emergente que afecta a todas las fases del proceso enseñanza aprendizaje. Es decir, sus extraordinarias posibilidades como medio de apoyo a la docencia se inician en el momento de la preparación de la instrucción al permitir en primer lugar, el acceso a fuentes de información próxima como las charlas electrónicas, las páginas Web y soportes digitales como los software educativos, estos últimos permiten transmitir una información tan compleja como se quiera y controlar el progreso de los educandos” (pp.345).

Por ello, se requiere prestar la atención adecuada a estos sistemas interactivos aplicados en la educación, darles el tratamiento que exige cualquier ambiente de 
aprendizaje considerando sus características particulares, los elementos que lo componen y el rol que juega cada uno de los actores educativos. La diferencia no la hace sólo la integración de la tecnología, sino el trabajo académico que se da para obtener todo el beneficio de esta integración en la educación.

\section{La Virtualidad de la Educación Superior y el Desempeño Docente}

El nuevo papel del profesor administrador de educación a distancia, es enseñar ya no sólo a aprender, sino a organizar la búsqueda de informaciones y a estructurarlas, este docente mediador de los procesos de aprendizaje se encuentra en una nueva legitimidad, en un pensamiento diferente de su acto pedagógico, por lo que se hace fundamental que sean capaces de asumir funciones basadas en:

a) Desarrollador de contenidos propios

b) Trabajo interdisciplinario

c) Utilización de la red como canal de comunicación.

d) Asumir las redes como espacio cooperativo y de formación.

e) Usar las redes como espacio de trabajo.

Las funciones del docente tradicional cambian cuando debe desarrollar sus actividades en un entorno virtual de Educación a Distancia, tomando en cuenta que deja de tener limitaciones geográficas, físicas, temporales y que tiende a dar respuesta a grupos de alumnos cada vez más heterogéneos y diversos (en el sentido más extenso de estas dos palabras), y por ello se debe redefinir su tarea profesional así como las funciones que deberá asumir en el desarrollo de ésta.

Es así como, la práctica educativa e instruccional de la educación virtual requiere con urgencia, un cambio en el quehacer docente que permita responder a las exigencias de estos tiempos, requiriendo a los administradores de educación bajo esta modalidad el reto de asumir un protagonismo ético, pedagógico y político, requeridos para adoptar características promotoras de la innovación y el cambio permanente en ellos y en los alumnos, desarrollando a estos actores en la dimensión humana, espiritual y pedagógica, comprometidos con el proceso de transformación política y social del país, enmarcados en los saberes cognitivos, procedimentales y actitudinales.

Esta actividad docente debe ser entendida como la persona encargada de mediar el aprendizaje de los alumnos. Hoy, con la incursión de las instituciones en la educación ofrecida bajo el entorno educativo, dentro de un nuevo marco relacional llamada entorno virtual de aprendizaje, la cual contempla la complementariedad del marco formativo clásico del aula con el uso más o menos intensivo de las tecnologías aplicadas a la educación, ya sea a través de los recursos de la red Internet, de materiales multimedia de aprendizaje o de espacios relacionales virtuales de aprendizaje.

Para alcanzar este propósito, es necesario enfrentar los cambios mediante un entrenamiento continuo, y reelaborando estructuras mentales, basadas en un compromiso con esta demandante modalidad educativa, en el cual los docentes funcionan como líderes conocedores de procesos que vinculen la aprehensión, asimilación y comprensión de conocimientos; más aún, cuando los nuevos hallazgos en la neurociencia, el desarrollo de la informática y la sofisticación de los sistemas de información, obliga a desmontar viejos esquemas, fundamentados en conceptos, creencias y técnicas que han ido quedando obsoletos.

De allí, que es necesario e imprescindible el cambio interno del docente para poder llegar a interactuar con el alumno de una manera eficiente en la construcción de aprendizajes significativos, donde se exige un docente mediador del aprendizaje, capaz de diseñar experiencias y propiciar situaciones que induzcan al crecimiento cognoscitivo, mediante la interacción directa con el medio que lo rodea. Para esto, el mediador debe detectar los bloqueos ambientales, emocionales y culturales, los resuelva y propicie una circunstancia tal que garantice éxito en el proceso.

Este educador debe estar actualizado en la exigencia de estos procesos de aprendizaje, en las estrategias de aprendizaje que le brindan al alumno la posibilidad de incrementar sus propias estrategias, con la utilización de recursos facilitadores de la aprehensión del conocimiento, los cuales posibilitan el organizar, relacionar y aplicar conocimientos, además de la seguridad de afrontar cualquier proceso despertando así la motivación e interés de los educandos a través de los materiales didácticos y estrategias de instrucción, a sabiendas que el docente junto a los materiales instruccionales son unos de los elementos básicos que conforman el proceso de aprendizaje en los sistemas de educación bajo la modalidad de estudios a distancia.

Los materiales instruccionales configuran el espacio de relación y mediación donde el estudiante construye su aprendizaje y donde la acción docente está en garantizar la prosecución de objetivos, bajo un grado optimo de calidad, fundamentado en los principios básicos del aprendizaje, propiciando actividades que faciliten el aprendizaje autodirigido, la motivación, la articulación entre la teoría y la práctica, a través del trabajo colaborativo y cooperativo y el manejo de múltiples representaciones del contenido, creando áreas de relación entre investigación, formación y trabajo. 
Es así como la autora teniendo, en cuenta todos los aspectos señalados anteriormente, plantea que el docente de entornos virtuales desarrollará una serie de funciones como:

a) Planificador $\mathbf{y}$ desarrollador de acciones formativas: para elaborar cursos en línea es imprescindible contar con un diseño bien organizado, el docente es el que tiene el control de su planificación, además de fomentar y fortalecer de manera eficiente aprendizajes significativos y el desarrollo autónomo del estudiante.

b) Desarrollador de contenidos: productor y evaluador de contenidos, integrándose en equipos interdisciplinares para la construcción de contenidos de manera consensuada y según los criterios de pares de especialistas de áreas comunes. Como desarrollador de contenidos, el docente debe ser poseedor de una visión constructivista del desarrollo curricular, capaz de convertir los materiales usados en su práctica presencial para entornos tecnológicos, además de ser promotores del cambio de los contenidos curriculares a partir de los avances de la sociedad que enmarca el proceso educativo.

Los contenidos deben ser especialmente diseñados para tal fin. Los autores deben adecuar el contenido para un medio donde se mezclan diferentes posibilidades de interacción de multimedios y donde la lectura lineal no es la normal. El usuario que lee páginas de Internet no lo hace como la lectura de un libro, sino que es más impaciente y escanea en el texto. Busca títulos, texto enfatizado en negrita o utilizando enlaces a otras páginas e imágenes o demostraciones. Si la información en la primera página implica scrolling o moverse hacia un lado diferente de su enfoque principal, es muy probable que el usuario se sienta desilusionado desde el principio del curso. "En estos ambientes los participantes del curso deben estar preparados para adoptar nuevos roles, seguir ciertas rutas con un objetivo específico en vez de navegar utilizando su propia ruta.

c) Administrador de Educación a Distancia: Utilizando los recursos tecnológicos, conocimiento las innovaciones y avances para aplicarlas en la administración de la instrucción, como administrador de este proceso el docente además, diagnostica necesidades académicas de los alumnos, acompaña al alumno, realiza monitoreo y supervisión de los avances del alumno retroalimentando su actuación.

Es así como, recibir los contenidos a través de un curso Web, es solo parte del proceso, también debe existir un mecanismo que permita la interacción y el intercambio de información. Es necesario que se tenga previsto un mecanismo de comunicación entre el participante y el facilitador o entre los participantes, para garantizar esta interacción. En la educación a distancia, donde el riesgo de deserción es muy alto, una de las maneras de evitarlo es haciendo que los participantes se sientan involucrados en la clase que están tomando, y acompañados por pares o por el facilitador. El monitoreo de la presencia del participante en la clase es importante para poder conocer si este visita regularmente el curso y si participa en el curso y con que frecuencia.

Por ello, el docente en la modalidad de estudios a distancia se convierte en un facilitador y promotor de aprendizajes, cumpliendo las funciones propias de: motivar y promover el interés de los participantes en el estudio de las temáticas propuestas, guiar y/o reorientar al alumno en el proceso de aprendizaje, evaluar el proceso y planificar las estrategias instruccionales y de evaluación entre otras, a sabiendas de que el tutor no es portador de contenidos, papel que en estos sistemas cumplen los materiales, sino un facilitador del aprendizaje.

Independientemente del medio que utilice con relación al alumno, el docente debe adecuarse a sus condiciones y circunstancias, discriminando las estrategias y recursos de acuerdo con los temas, asignaturas o cursos y sus necesidades y dificultades; estando a su disposición durante su proceso de aprendizaje. La modalidad de estudios virtuales está fundamentada en principios del aprendizaje que hacen hincapié en actividades que faciliten el aprendizaje autodirigido, la motivación, la acción, la responsabilidad, la contextualización de los contenidos en la vida real, la articulación entre teoría y práctica, la realización de actividades genuinas, el trabajo de tipo colaborativo y cooperativo, las interacciones sociales, la variedad de conocimientos, las múltiples representaciones del contenido y la reflexión, entre otros.

En este sentido, el docente debe manejar diferentes estrategias que le permitan hacer llegar el contenido del curso de una forma más efectiva y eficaz, tomando en cuenta para el diseño de las mismas la perspectiva del alumno, del facilitador y del facilitador-alumno, a fin de lograr detallar las actividades que cada miembro del curso realizará y la participación del facilitador en el monitoreo de estas estrategias.

La facilitación en la modalidad de estudios virtuales debe ser liderada por el especialista en la materia calificado y capacitado específicamente en la facilitación de procesos de aprendizajes en línea. Esta es una habilidad que se desarrolla, para esa función no es 
suficiente buscar al mejor profesor de la asignatura presencial, ya que está demostrado según lo plantea Haavind (2000), que:

Las estrategias de facilitación que son aplicadas efectivamente en clases presenciales, tienen efectos no positivos en la educación a distancia. El facilitador efectivo utiliza estrategias que para estimular la colaboración entre estudiantes y para guiar el proceso hacia lo que es importante para el desarrollo del contenido. En los Chat y foros el facilitador debe monitorear todas las discusiones que se generan en lo sitios de discusión telemáticos y responder en breve tiempo las interrogantes y los correos e inquietudes de los participantes.( pp. 64)

Para entender el papel docente virtual, se plantea la descripción de dos las tareas básicas del docente virtual, con la intención de definir luego las competencias en términos generales, a saber:

a) Tarea académica. Asesora al estudiante sobre los aspectos directamente relacionados con el estudio y aprendizaje de los contenidos del curso:

- Manejo de información. Proporciona explicaciones al estudiante sobre cómo utilizar las diferentes fuentes de información, en la forma de lecturas, sonidos, imágenes y sitios de Internet. Un logro adicional es la motivación del participante.

- Construcción del conocimiento. Propicia la reflexión, presenta ejemplos y propone soluciones, por ejemplo a través de intercambios en foros, por correo electrónico, en trabajos grupales o en parejas.

b) Tarea orientadora. El docente orienta al participante para resolver problema, así como su acceso y socialización

El rol del facilitador en la utilización de estrategias pertinentes para la modalidad de estudios virtuales, trae consigo un alto nivel de motivación de los participantes, a través del trabajo fuerte y ético, ofreciendo además el soporte académico y técnico como medidas que generalmente producen buenos resultados. El soporte del facilitador a los participantes se define no sólo como el proporcionar actividades académicas, sino también la identificación y solución de problemas, proveer oportunidades para interacción entre los participantes de un curso con el facilitador, y la habilidad de mantenerlos motivados a través del seguimiento de los logros alcanzados y la retroalimentación. Por lo que la interacción entre grupos colaborativos de trabajo generalmente produce resultados positivos en los participantes de esta modalidad de estudios.

Es así como, el tutor facilitador de la modalidad de Estudios Virtuales, debe recurrir a diferentes estrategias instruccionales cumpliendo con una serie de principios. A continuación se muestran algunos de ellos basados en lo planteado por Torres (2003):

a) Activar los procesos cognitivos, para aplicar este principio el facilitador debe: Dar soporte y guía al participante durante el desarrollo de la asignatura o curso, requerir: comparaciones, clasificaciones, inducciones, deducciones, análisis de errores, construcciones, abstracciones, análisis, metáforas, explicaciones, productos, en otras palabras, la participación activa, desarrollar los contenidos desde diferentes perspectivas, utilizar vínculos a unidades de información que ofrezcan flexibilidad, interactividad, posibilidad de acceso a variadas fuentes de información. utilizar esquemas conceptuales.

b) Promover interacción social, participación, ofreciendo al participante múltiples formas de comunicación: correos, “chats”, foros, pizarrón, tele-conferencia, vídeos, audio, audio-conferencia, multimedia, actividades de ayuda, elaboración de proyectos, formulación de preguntas, tormenta de ideas, pequeños grupos de discusión, estudio de casos, juego de roles, asignación de lecturas e investigaciones, presentación de invitados (audio, vídeos, lecturas), trabajos de campo, exámenes, prácticas, juegos, problemas, portafolios, simulaciones, tutorías, y retroalimentar, anunciar las expectativas, explicitar cómo serán las interacciones y las responsabilidades. generar productos de las actividades realizadas. permitir a los estudiantes seleccionar problemas de interés para su estudio, conformar grupos pequeños de discusión y de trabajos. solicitar a los estudiantes resúmenes de cada discusión. centrarse en la realización de tareas específicas y valiosas. favorecer el desarrollo de interacciones académicas tipo diálogo, en donde el significado se construya compartiendo y no se pierda la calidez humana

c) Ayudar al aprendizaje auto-dirigido, el facilitador debe promover la ejercitación de los contenidos a través del manejo del tiempo, el planteamiento de objetivos y la autoevaluación, ayudar a tomar riesgos, dar 
soporte, retroalimentación, aconsejar, monitorear, propiciar la elaboración de trabajos en grupo con metas libres.

d) Capturar, mantener y estimular el interés, enfatizando en la experimentación y resolución de problemas, el descubrimiento y construcción de nuevos conocimientos, animar a los participantes a pensar y buscar información para realizar los trabajos desde diferentes perspectivas, publicar los mejores trabajos y elogiarlos públicamente, aumentar los niveles de dificultad como una manera de ofrecerle retos al estudiante. (pp.78)

Como lo plantea la autora antes señalada, estos principios permiten ubicar muchas de las estrategias y/o recursos que el facilitador podría implementar de múltiples maneras en la práctica de la administración de su curso a distancia. Todo dependerá, de la situación o contexto en que ocurra el proceso y a su vez, de las respuestas que se puedan ofrecer a múltiples interrogantes, como por ejemplo: qué es aprender y para qué enseñar.

Es así como, los docentes que participan como administradores de procesos instruccionales a distancia requiere del mejoramiento de habilidades que ya poseen, más que del desarrollo de habilidades nuevas, por lo que deben tomar en cuenta los siguientes aspectos:

a) Evaluar el contenido que puede incluirse y abarcarse efectivamente en cualquier curso. Cuidar que no exista repitencia de contenidos y manejo de información innecesaria. El facilitador tendría que producir sus contenidos y adecuar las estrategias y recursos a la modalidad de estudios. Por ello, se debe revisar los contenidos a exponer en un curso a distancia, ya que al presentar el mismo contenido de un curso presencial a distancia usualmente requiere más tiempo y de mas formas y maneras de presentarlo, esta definición de los contenidos debe ser realizada en forma consensuada con otros docentes de la cátedra, de tal manera que se manejen criterios unificados y los contenidos puedan ser trabajados por el resto de los profesores de la materia, además de garantizar su durabilidad en el tiempo.

b) Tomar en cuenta las características de los participantes, probablemente tendrán estilos de aprendizaje diferentes. Algunos se les facilitará aprender en equipo, mientras que otros serán excelentes trabajando independientemente.

c) Diversificar y programar las actividades y evitar las lecturas largas. Se deben mezclar presentaciones del contenido con discusiones, análisis, analogías, foros, ejercicios, ejemplos y estudios de casos locales de los estudiantes como sea posible, de esta manera se propiciaría la labor social de los participantes en actividades inherentes de su localidad, aplicando en su medio ambiente los conocimientos del curso, utilizando las herramientas tecnológicas con que se cuente y las ventajas que ofrezcan.

d) Diseñar guías didácticas que complementen la información presentada en el curso, esta opción va a ser esencial como elemento motivador, que sirva para suscitar el interés del participante al cual se le van a presentar distintas estrategia que el equipo docente decida incorporar en la guía, además de contenido mas extenso que enriquezca aún más su conocimiento. Más allá del plano motivacional pero estrechamente relacionado con él, esta guía permite estructurar tanto la presentación de contenidos como el tipo de interacción didáctica con los alumnos de la asignatura.

e) Diseñar estrategias que permitan el reforzamiento de la información, para revisión, repetición y remedio. Para esto, las asesorías telefónicas, el correo electrónico o la audioconferencia pueden ser muy útiles. Para ello, se hace necesaria la utilización de actividades cortas, comprensivas y concisas, preguntando las cosas directamente. Teniendo presente que las distancias hacen más lentas las comunicaciones y que los alumnos necesitarán más tiempo para responder. Además de lo antes planteado, esta estrategia permitirá al participante investigar, prepararse y fundamentar su participación en los encuentros, además de propiciar el pensamiento crítico y la participación bien fundamentada de los estudiantes.

Si el facilitador utiliza estrategias adecuadas en la administración de su curso podrá identificar y satisfacer las necesidades e inquietudes de los participantes, al mismo tiempo que podrá establecer mecanismos adecuados que le permitan mejorar y evaluar constantemente el curso, mejorando así la interacción y la retroalimentación

De tal manera que el desempeño docente en esta modalidad de estudios es sumamente importante para lograr su éxito, ya que es quien deberá asumir una actitud investigadora y crítica, ser conocedor del uso y alcances de las nuevas tecnologías de la información y la comunicación, de la filosofía y objetivos de la educación a distancia, y lo que es más importante, experto y conocedor de la teoría andragogica que fundamenta esta modalidad de estudios.

El papel que tienen los docentes en esta actuación en la educación a distancia es estelar. La formación de los profesores en el dominio de este método de educación, ha de 
sobrepasar el conocimiento de los contenidos, procesos y habilidades para alcanzar las barreras de un nuevo proyecto educativo a gran escala. En las investigaciones de Bricall, (2000), ha salido a relucir que "...la introducción de las nuevas tecnologías en la educación no supone la desaparición del profesor, aunque obliga a establecer un nuevo equilibrio en sus funciones". Y es la propia forma de enseñar, la metodología docente, la que ha de cambiar: más centrada en el estudiante, más orientada al mundo y las necesidades profesionales, más orientadas al nuevo papel del profesor.

\section{EL DOCENTE DE EDUCACIÓN A DISTANCIA Y EL PROCESO DE EVALUACIÓN DE LOS APRENDIZAJES EN LÍNEA.}

En la modalidad de educación a distancia se hace referencia específicamente al auto aprendizaje. En esta propuesta de autogestión, la evaluación se presenta como participativa y con soporte comunicacional. La no presencialidad en los entornos de educación a distancia obligan a planificar estrategias de evaluación que respondan a la modalidad de estudios, cuidando la efectividad de los procesos de evaluación, partiendo del hecho que las técnicas presenciales dejan de tener aplicabilidad en este sistema, deben diseñarse otras de alternativas relacionadas claramente con los procesos de autogestión de la formación y de responsabilidad del propio sujeto.

Es importante señalar que las estrategias de evaluación en esta modalidad requieren que tanto los profesores como los estudiantes desarrollen habilidades y competencias que permitan aprovechar estas herramientas y mejorar su efectividad. El docente debe evaluar de una forma continua durante todo el proceso de aprendizaje, manteniendo así el seguimiento de su desarrollo. Para ello se generan espacios de autoevaluación con un alto grado de coherencia. Simultáneamente se integran lo cualitativo y cuantitativo; obligatorias y optativas; individuales y grupales

\section{$\mathrm{Al}$ respecto, Rubio (2005), plantea que:}

En educación a distancia el facilitador debe tener presente que la evaluación es tomada de dos formas: la primera de ellas como estrategia de aprendizaje, la segunda como medio de acreditación de conocimientos. En cualquier caso, la tecnología aplicada a la evaluación permite personalizar el proceso y potenciar las habilidades del estudiante. Son muy usuales las autoevaluaciones interactivas, en las que la retroalimentación argumentada e inmediata es muy importante y útil para que el estudiante pueda conocer el nivel alcanzado y a la vez corregir y conocer las partes del contenido en las que debe centrar más su atención. (p.214).
La evaluación de los aprendizajes bajo la modalidad de estudios virtuales, presenta dos direcciones:

a) Investigación: Se motiva al estudiante a la investigación, al trabajo independiente y grupal, donde ponga en práctica los conocimientos adquiridos durante el desarrollo de los objetivos y contenidos planteados.

b) Trabajo de campo: El estudiante realiza trabajos a distancia con reconocimiento académico, demostrando el dominio de contenidos y la aplicación en la práctica de casos concretos.

Esta última es considerada como el medio más fundamental de control de rendimiento académico de los alumnos e instrumento primordial de calificación del docente.

La evaluación en la educación a distancia es asumida al igual que en la educación presencial, es un proceso que se realiza al inicio, durante y al final de los procesos de enseñanza y aprendizaje. Al igual que en una modalidad presencial el docente debe evaluar en función de los momentos de evaluación diagnóstica, formativa y sumativa. La evaluación diagnóstica es fundamental en el comienzo del curso o carrera, para determinar los conocimientos del estudiante.La evaluación formativa está constituida por las actividades propuestas durante el proceso de aprendizaje, los resultados de estas retroalimentan a los estudiantes e informan al sistema permitiendo el mejoramiento continuo. La evaluación sumativa es la que cierra parcial o totalmente el proceso permitiendo la certificación de aprobado o reprobado durante el proceso.

Es así como el docente debe asumir la evaluación como un debe ser un proceso de diagnóstico, diálogo, comprensión, retroalimentación constante de los logros de aprendizajes.

Como lo señala Barbera (2004 pp.45) los docentes en esta modalidad de estudios debe tomar en cuenta los siguientes principios para la planificación de la evolución:

a) Coherencia entre el programa evaluativo y el proceso de enseñanza aprendizaje

b) Potenciación de una evaluación significativa para el alumno y para la materia 
disciplina.

c) Claridad y transparencia en la comunicación y negociación de objetivos, referentes y criterios de evaluación.

d) Incorporación de itinerarios de evaluación que contemplen tareas auténticas.

e) Participación de los estudiantes y mayor relación profesor / estudiante.

f) Inclusión de tareas de evaluación que generen capacidad de reflexión y toma de decisiones consciente.

g) Integración de procesos compartidos de comunicación y aprovechamiento de los resultados de la evaluación.

h) Realización de metaevaluaciones que incorporen los comentarios argumentados de los alumnos y de otros profesores.

A la luz de lo anteriormente expuesto, la evaluación en la educación a distancia, constituye un elemento fundamental del proceso instruccional y es en este proceso donde el docente haciendo uso de las tecnologías disponibles, establece estrategias que les permite abordar desde diferentes perspectivas los procesos de análisis, diseño, producción, ejecución y evaluación de los estudiantes, tomando en cuenta que su planificación debe ser abordada desde el consenso y estudio minucioso, logrando estrategias de evaluación fluidas y precisas, a continuación se presentan las mas utilizadas:.

- La retroalimentación como estrategia de formación

- El Chat como estrategia de formación formativa, interactiva e inmediata que favorece la reflexión.

- El foro como estrategia de evaluación sumativa o formativa

- Video permitiendo al estudiante observar, autoevaluar y coevaluar

- Trabajos escritos individuales o grupales, enviados por medio del correo electrónico
- Análisis de casos

- Evaluaciones individuales estructuradas o de desarrollo

- Participación activa en el desarrollo del curso

Estas estrategias brindan autonomía y una estructura del proceso evaluativo que se construye en base a la negociación y el diálogo, estableciendo compromisos pedagógicos que posibiliten el logro de los aprendizajes del estudiante a distancia, además el docente podrá formular los criterios de lo que se quiere evaluar y pretende evaluar en cada una de las actividades. El docente debe tomar en cuenta las características de la cátedra, para planificar las estrategias didácticas, contenidos, evaluaciones, autoevaluaciones y todos los elementos que intervienen en un proceso instruccional.

\section{Registro de resultados de evaluación}

Según lo planteado por Bravo (2000), toda actividad formativa o sumativa reconocida para propósitos de evaluación debe incluir instrumentos, con criterios definidos de evaluación, además de un registro de las asesorías, seguimiento y evaluación de logros. Los modelos varían dependiendo de los seleccionados por el facilitador, estos no son una receta, lo interesante es llevar el record al participante de sus actuaciones, propiciar la participación y retroalimentar el proceso de aprendizaje del alumno.

En resumen la evaluación de los aprendizajes en la educación a distancia proporciona la posibilidad de monitoreo, seguimiento y evaluación de los logros de aprendizajes, dando continuidad al proceso con la inmediatez con que se puede dar respuesta a las necesidades del estudiante, por medio de una retroalimentación oportuna que permite brindarle las orientaciones que este necesita para alcanzar el éxito, facilitando el desarrollo de habilidades, propiciando el contacto permanente, apoyando un aprendizaje activo que favorece la obtención de los logros en el aprendizaje.

\section{GESTIÓN DOCENTE EN LA ADMINISTRACIÓN DE EDUCACIÓN A DISTANCIA}

Es evidente que el docente administrador de educación a distancia tendrá que redefinir su rol respecto al asumido en el sistema de enseñanza convencional. Las instituciones están en constante cambio que le demanda la sociedad actual y por ende sus docentes deben estar preparados para el abordaje e inserción en esos cambios. Es así como la figura 
omnipresente del profesor deja paso al trabajo coordinado de los integrantes de una cátedra que impartirán cursos Web de su asignatura.

Es por ello, que la gestión del docente presencial cambian cuando este administra actividades de educación bajo un entorno virtual, asumida esta como una modalidad de estudios donde no existen limitaciones geográficas, físicas, temporales y que tiende a dar respuesta a grupos de alumnos cada vez más heterogéneos y diversos. Partiendo del hecho que la función del docente se redefine, asumiendo competencias para el desarrollo de esta modalidad de estudios.

Es así como, partiendo del hecho que el docente de esta modalidad de educación a distancia, debe estar capacitado en el conocimiento de las características de los entornos tecnológicos, colocándose en la perspectiva del análisis de potencialidades de ayuda o andamiaje didáctico que ofrecen las tecnologías de la información y la comunicación, tomando en cuenta la necesidad de desarrollar una relación entre pedagogía, andragogía y tecnología, para la concepción de un profesorado que comprenda lo que significa enseñar con compromiso en la modalidad de educación a distancia.

Por ello, la función docente se ve desde la perspectiva de una formación integral, que le permita a estos sujetos primero la comprensión y después la apropiación de la virtualidad en su quehacer docente, a sabiendas de que en gran medida depende de ellos el éxito o fracaso de la administración de cursos bajo la modalidad virtual, de allí la gran importancia de poseer las competencias que responda a las exigencias de la modalidad y a las necesidades que actualmente existe por la demanda nacional, donde se pretende dar solución a los requerimientos futuros de educación continua y de accesibilidad al sistema de Educación Superior en nuestro país.

Es así como, la gestión del docente debe estar inmersa en unas competencias que debe tener, estas a objeto de este estudio han sido contextualizadas en el plano pedagógico como la expresión didáctica de la profesión, a partir del concepto de las habilidades generalizadas, los núcleos de conocimientos y los valores profesionales que le permiten al docente actuar de manera creativa, pertinente, efectiva y eficaz en el diseño de contenidos, la administración de los aprendizajes y los procesos de evaluación y retroalimentación del proceso instruccional y de los logros de los alumnos.

En primera instancia, gestión de docente diseñador y productor de contenido, teniendo la responsabilidad de desarrollar con criterios de originalidad el tratamiento instruccional y producción de contenidos de su curso asignatura para la Web, para su abordaje se han diseñado los siguientes indicadores de logro:

Indicador Actitudinal: Aborda la producción de los contenidos con base en las necesidades y características de la asignatura

Indicador Cognoscitivo: Identifica los componentes centrales de un curso Web y los contextualiza dependiendo de sus características

\section{Indicadores Procedimentales:}

1. Desarrolla los contenidos del curso, indicando la ruta formativa, su plan de desarrollo, el material de apoyo a la formación y las estrategias que permitan la mediación pedagógica

2. Aplica principios de las teorías de instrucción y aprendizaje para el diseño de contenidos

3. Aplica los estándares de diseño instruccional para la administración de cursos virtuales o en línea

En relación a esta gestión y para garantizar el diseño y efectividad de los contenidos didácticos, es fundamental la selección de materiales, estrategias instruccionales y de evaluación, los cuales deben estar muy bien definidos en el diseño instruccional previo.

Aunada a lo anterior y como otra gestión de suma importancia se presenta la gestión del docente administrador de cursos Web, teniendo como competencia el utilizar las herramientas que ofrecen las Tecnologías de la Información y la Comunicación para la administración de cursos bajo la modalidad de estudios a distancia, con sentido ético y responsabilidad social, planteándose los siguientes indicadores de logro para su abordaje

Indicador Actitudinal: Asume las Tecnologías de la Información y la Comunicación dentro del marco de la administración de Educación a Distancia

Indicador Cognoscitivo: Conceptualiza la formación a distancia como un proceso complejo, teniendo en cuenta las TIC como herramienta y medio de instrucción. 
Indicador Procedimental: Integra diferentes estrategias instruccionales para la administración de instrucción a distancia, respondiendo a las características del participante y del curso

Esta gestión será desarrollada en espacios tecnológicos y presenciales dependiendo las características de los cursos, apoyados por estrategias y recursos que le permitan garantizar la eficacia del proceso.

Por último, se plantea la gestión del docente orientador mediador de aprendizajes capaz de articular las herramientas necesarias para "enseñar”, "guiar” y "evaluar” a los participantes, también ampliar, profundizar y relacionar con otros temas, en caso de que esto sea solicitado por el participante o si él mismo lo considere pertinente, con capacidad para aclarar las dudas, proponer nuevos ejemplos y resolver las problemáticas que los participantes presenten, tal como haría en la modalidad presencial, valorando al estudiante como centro del proceso instruccional con base en los lineamientos curriculares y las actividades programadas para su desarrollo.

Para poder medir el logro de esta gestión se plantean los siguientes indicadores, desglosados por los saberes, Ser referidos a los logros actitudinales, saber relacionados al abordaje de los conocimientos y el saber hacer que es la aplicabilidad de ese conocimiento, lo procedimental, a continuación se detallan:

Indicador Actitudinal: Asume su compromiso como mediador de la instrucción, tomando en cuenta el ritmo de aprendizaje de los estudiantes y los criterios institucionales

Indicador Cognoscitivo: Identifica las evidencias de aprendizajes de sus alumnos, las estrategias instruccionales individuales, grupales y de facilitacion, los recursos y las estrategias de evaluación que conlleven al logro de los indicadores que se aplican con base a una determinado conocimiento.

Indicador Procedimental: Asesora al estudiante sobre los aspectos directamente relacionados con el estudio y aprendizaje de los contenidos del curso y lo retroalimenta en cuanto al logro de los indicadores planteados en el desarrollo del curso Web

El docente de esta modalidad de estudios estimula y asegura la calidad de los aprendizajes, la satisfacción de los estudiantes, basados en el estrategias que estimulan y animan a los estudiantes a participar, en línea y fuera de línea. Como por ejemplo, los portafolios, para estimular a los estudiantes a que desarrollen competencias comunicativas escritas y reflexivas, promoviendo la construcción de comunidades de aprendizaje colaborativo con diferentes "backgrouds" cultural, histórico a través de la integración de las tecnologías emergentes para la transformación del proceso de enseñanza

La gestión del docente de la modalidad a distancia en este estudio queda definido con las siguientes funciones: diseñador y productor de contenidos, motivar y promover el interés de los participantes en el estudio de las temáticas propuestas, guiar y/o reorientar al alumno en el proceso de aprendizaje atendiendo a sus dudas o dificultades, ampliar la información, evaluar el proceso de aprendizaje, diseñar la instrucción de su curso y las evaluaciones de aprendizaje.

\section{REFLEXIONES FINALES}

La educación a distancia requiere de docentes capacitados con destrezas y una exigente preparación específica para enfrentar el tratamiento del proceso instruccional en esta modalidad de estudios, tomando en cuenta la capacidad cognoscitiva de los alumnos como sujetos de conocimiento.

El docente que utiliza las herramientas que ofrecen las TIC y aplica estrategias instruccionales que promueva el análisis, el trabajo socializado, colaborativo, la participación activa, debe aprovechar el amplio abanico de posibilidades sin perder de vista los objetivos educativos y los indicadores planteados para lograr en su curso, y cumplir efectivamente una función "mediadora y facilitadora". Y gracias a esa función el facilitador será más libre al momento de realizar sus planificaciones de clases, además de poder dedicarse a la verdadera acción docente: la de relacionarse con sus alumnos en el nivel más humano, más profundo y formativo, de persona a persona.

Las tecnologías de la información y comunicación utilizadas racionalmente darán pie a una formación humana más flexible, coherente y autónoma, porque actualmente se desea un sistema integralmente abierto. Al carácter selectivo tan acusado de la modalidad presencial, debe suceder una orientación flexible, siempre ágil y provisional; una formación que 
nunca se considere definitiva donde siempre se involucre la función institucional, la función docente y la función de los participantes para que se logre el aprendizaje y el trabajo creativo.

El docente será cada vez más un facilitador, un orientador de trabajos y de líneas formativas, será el auténtico mentor que acompañará al alumnado en su camino de formación, un camino que él debe recorrer activa y libremente con la asesoria permanente del docente a través de la utilización de las herramientas que ofrecen las tecnologías de la información y la comunicación y la red Internet.

El docente es el ente responsable de prestar la atención adecuada a los aspectos que involucra esta modalidad de estudios considerando sus características particulares y los elementos que lo componen, así como, el rol que juega cada uno de los actores educativos, partiendo del hecho que el alumno es el centro del proceso instruccional.

Un docente comprometido con la educación deberá actuar preparando a las nuevas generaciones para convivir con los medios. No se puede seguir enseñando a las generaciones del futuro con las herramientas que formaron parte de nuestro pasado.

\section{REFERENCIAS}

Asencio, S. (2002) Desafíos y fundamentos de la educación virtual. Bolivia

Bates, A (1999) La Tecnología en la Enseñanza Abierta y la Educación a Distancia. Editorial Trillas. México.

Barberà, E (2004). La evaluación escrita en el área matemática: contenido y tendencias. Anuario de Psicología,.

Bravo, JL. (2000) Los Sistemas Interactivos en la Docencia Universitaria. Santiago de Compostela
Bravo, JL. (2005) “Multimedia educativo” Comunicación y Pedagogía.

Bricall, J., (2000). "Informe Universidad 2000", [Documento en En línea], http://www.crue.org/cap7.pdf. Barcelona - España

Fainholc, B, (2000) Formación del Profesorado para el Nuevo Siglo. Aportes de la Tecnología Educativa Apropiada, Lumen Humanitas, Buenos Aires.

García A, L. (2001). La Educación a Distancia: de la Teoría a la Práctica. Barcelona: España Ariel Educación.

García Aretio, L. (2007). La educación a distancia. Madrid: UNED España

Haavind, S (2000). Why Don t Face to Face teacher strategies work in the virtual classroom.

Inciarte, M (2007) Diseño Instruccional para Educación a Distancia. Sistema de Educación a Distancia Universidad del Zulia.

JiméneZ, B.; GonzáleZ, A-P. y GisberT, M. (1997): El papel del profesor ante el reto de las Nuevas Tecnologías. En ALONSO, C. (Coord. ): La ecnología Educativa a finales del siglo $X X$ : Concepciones, conexiones y límites con otras disciplinas. EUMO. Barcelona.

Quevedo, M (2000) Proyectos de educación a distancia en Venezuela. Caracas: Universidad Central de Venezuela

Ramírez, A (2000). Diseño de un Interfaz para Ambientes Colaborativos. Fondo Editorial EAFIT. Medellín.

Rosario, J.,(2006), Los Weblogs como Incentivo a la Lectura y el Aprendizaje en los Centros Educativos, (p. 14), Instituto FIEC, España, ISBN: 84-93515-2-0.

Rubio, M., (2005). Proceso de autoevaluación de los programas de educación a distancia basado en el proyecto "Centro Virtual Para el Desarrollo de Estándares de Calidad Para la Educación Superior a Distancia en América latina y El Caribe: Documento Introductorio. Loja, Ecuador: Universidad Técnica Particular de Loja

Silvio, J (2000). La Virtualización de la Universidad. Colección Respuestos. Ediciones IESALC / UNESCO. Caracas. 
Silvio, J. (2000) La virtualización de la universidad. ¿Cómo podemos transformar la educación superior con la tecnología?. Caracas: Ediciones IESALC/UNESCO

Torres de I, M. (2003). Teorías del aprendizaje y de instrucción aplicadas al diseño de cursos Web. Tesis Doctoral. URBE. Maracaibo, Edo. Zulia

Zubiria S.J.,(1994), Los modelos pedagógicos, Santa Fe de Bogotá, D.C., FAMDI,. 